\title{
Convex-Ear Decompositions and the Flag h-Vector
}

\author{
Jay Schweig \\ University of Kansas \\ Kansas, U.S.A. \\ jschweig@math.ku.edu
}

Submitted: Jun 10, 2010; Accepted: Dec 13, 2010; Published: Jan 5, 2011

Mathematics Subject Classification: 05E45, 06F30, 52B22

\begin{abstract}
We prove a theorem allowing us to find convex-ear decompositions for rankselected subposets of posets that are unions of Boolean sublattices in a coherent fashion. We then apply this theorem to geometric lattices and face posets of shellable complexes, obtaining new inequalities for their h-vectors. Finally, we use the latter decomposition to give a new interpretation to inequalities satisfied by the flag hvectors of face posets of Cohen-Macaulay complexes.
\end{abstract}

\section{Introduction}

The f-vector of a finite simplicial complex $\Delta$, which counts the number of faces of the complex in each dimension, is arguably its most fundamental invariant. The h-vector of $\Delta$ is the image of its f-vector under an invertible transformation. Somewhat surprisingly, properties of a complex's f-vector are sometimes better expressed through its h-vector. A good example of this phenomenon are the Dehn-Sommerville relations (see, for instance, [17]), which state that the h-vector of a simplicial polytope boundary is symmetric.

The main complexes we study in this paper are all order complexes, namely complexes whose simplices correspond to chains in posets. Since a poset and its order complex hold the same information, we often refer to them interchangeably. E.g., we may speak of the facets or h-vector of a poset, or to a chain in an order complex.

Convex-ear decompositions were first introduced by Chari in [3]. Heuristically, a complex admits a convex-ear decomposition if it is a union of simplicial polytope boundaries which fit together coherently (see Definition 2.3). Suppose a $(d-1)$-dimensional complex $\Delta$ admits such a decomposition. In [3], Chari shows that the h-vector $\left(h_{0}, h_{1}, \ldots, h_{d}\right)$ of $\Delta$ satisfies, for $i<d / 2, h_{i} \leq h_{i+1}$ and $h_{i} \leq h_{d-i}$. In [15], Swartz shows that $\Delta$ is 2-CM, and that $\left(h_{0}, h_{1}-h_{0}, h_{2}-h_{1}, \ldots, h_{\lfloor d / 2\rfloor}-h_{\lfloor d / 2\rfloor-1}\right)$ is an M-vector (called an $O$-sequence by some authors). Convex-ear decompositions have proven quite useful, as they have been applied to coloop-free matroid complexes [3], geometric lattices [9], coloring complexes [6], 
$d$-divisible partition lattices [16], coset lattices of relatively complemented finite groups [16], and finite buildings [15].

In [11], we find a convex-ear decomposition for rank-selected subposets of supersolvable lattices with nowhere-zero Möbius functions. In the process, we obtain a decomposition for order complexes of rank-selected subposets of the Boolean lattice $B_{d}$. In this paper we build upon this result with Theorem 3.1, which gives convex-ear decompositions for rankselected subposets of posets that are unions of Boolean lattices, pieced together nicely. In Section 3 we recall several useful results from [11], and then prove this theorem.

Sections 4 and 5 are devoted to the applications of Theorem 3.1 to geometric lattices and face posets of shellable complexes, respectively. Taken together, these results (along with those of Chari and Swartz) give us the following.

Theorem 1.1. Let $\Delta$ be the order complex of a rank-selected subposet of either

1: a geometric lattice, or

2: the face poset of the codimension-1 skeleton of a Cohen-Macaulay complex.

Then $\Delta$ is 2-CM and its $h$-vector $\left(h_{0}, h_{1}, \ldots, h_{d}\right)$ satisfies, for all $i<d / 2, h_{i} \leq h_{i+1}$ and $h_{i} \leq h_{d-i}$. Moreover, $\left(h_{0}, h_{1}-h_{0}, h_{2}-h_{1}, \ldots, h_{\lfloor d / 2\rfloor}-h_{\lfloor d / 2\rfloor-1}\right)$ is an M-vector.

For item 2 above, we use the fact that the set of h-vectors in question remains unchanged if we replace the Cohen-Macaulay requirement with the stronger condition of shellability (see the proof of Theorem 6.2).

Finally, in Section 6, we use the decomposition from Section 5 and techniques similar to those in [9] to prove that the flag h-vector $\left\{h_{S}\right\}$ of a Cohen-Macaulay complex's face poset satisfies $h_{T} \leq h_{S}$ whenever $S$ dominates $T$ (in the sense of Definition 2.10).

\section{Preliminaries}

We assume a familiarity with simplicial complexes and partially ordered sets (see [14]). All our simplicial complexes will be finite and pure.

The $f$-vector of a $(d-1)$-dimensional simplicial complex $\Delta$ is the integral sequence $\left(f_{0}, f_{1}, \ldots, f_{d}\right)$, where $f_{i}$ counts the number of $(i-1)$-dimensional faces of $\Delta$. The $h$-vector of $\Delta$ is the sequence $\left(h_{0}, h_{1}, \ldots, h_{d}\right)$ given by

$$
\sum_{i=0}^{d} f_{i}(t-1)^{d-i}=\sum_{i=0}^{d} h_{i} t^{d-i}
$$

We use the following alternate definition of shellability, easily seen to be equivalent to the standard one (see [1]).

Definition 2.1. Let $F_{1}, F_{2}, \ldots, F_{t}$ be an ordering of the facets of $\Delta$. This ordering is a shelling if and only if for all $j<k$ there exists a $j^{\prime}<k$ satisfying

$$
F_{j} \cap F_{k} \subseteq F_{j^{\prime}} \cap F_{k}=F_{j^{\prime}}-x
$$

for some element $x$ of $F_{j^{\prime}}$. 
We also use the following result of Danaraj and Klee for showing that a given complex is a ball.

Theorem $2.2([4])$. Let $\Delta$ be a full-dimensional shellable proper subcomplex of a sphere. Then $\Delta$ is a ball.

\subsection{Convex-ear decompositions}

Definition 2.3. Let $\Delta$ be a $(d-1)$-dimensional complex. We say $\Delta$ admits a convex-ear decomposition, or c.e.d., if there exists a sequence of pure $(d-1)$-dimensional subcomplexes $\Sigma_{1}, \Sigma_{2}, \ldots, \Sigma_{t}$ such that

$i: \bigcup_{i=1}^{t} \Sigma_{i}=\Delta$.

ii: For $i>1, \Sigma_{i}$ is a proper subcomplex of the boundary complex of a d-dimensional simplicial polytope, while $\Sigma_{1}$ is the boundary complex of a d-dimensional simplicial polytope.

iii: Each $\Sigma_{i}$, for $i>1$ is a topological ball.

iv: For $i>1, \Sigma_{i} \cap\left(\bigcup_{j=1}^{i-1} \Sigma_{j}\right)=\partial \Sigma_{i}$.

Convex-ear decompositions were introduced by Chari in [3], where the following was proven.

Theorem 2.4. When $\Delta$ admits a convex-ear decomposition its h-vector satisfies, for all $i \leq\lfloor d / 2\rfloor$,

1: $h_{i} \leq h_{i+1}$ and

2: $h_{i} \leq h_{d-i}$.

In [15], Swartz proved the following analogue of the g-Theorem for complexes with convex-ear decompositions. An M-vector is the degree sequence of an order ideal of monomials.

Theorem 2.5. Let $\Delta$ be a complex admitting a convex-ear decomposition, with h-vector $\left(h_{0}, h_{1}, \ldots, h_{d}\right)$. Then the vector

$$
\left(h_{0}, h_{1}-h_{0}, h_{2}-h_{1}, \ldots, h_{\lfloor d / 2\rfloor}-h_{\lfloor d / 2\rfloor-1}\right)
$$

is an M-vector. Furthermore, $\Delta$ is 2-CM. 


\subsection{Order complexes and flag vectors}

Recall that the order complex of a poset $P$, which we write as $\Delta(P)$, is the simplicial complex whose faces are chains in $P$. If $P$ has a unique minimal element $\hat{0}$ or a unique maximal element $\hat{1}$, we do not include these in the order complex. That is, simplices in $\Delta(P)$ are chains in $P-\{\hat{0}, \hat{1}\}$. All our posets are ranked.

For the remainder of this section, let $P$ be a rank- $d$ poset with a $\hat{0}$ and $\hat{1}$. A labeling of $P$ is a function $\lambda:\left\{(x, y) \in P^{2}: y\right.$ covers $\left.x\right\} \rightarrow \mathbb{Z}$. For a saturated chain

$$
\mathbf{c}:=x=x_{0}<x_{1}<\cdots<x_{t}=y
$$

the $\lambda$-label of $\mathbf{c}$, written $\lambda(\mathbf{c})$, is the word

$$
\lambda\left(x_{0}, x_{1}\right) \lambda\left(x_{1}, x_{2}\right) \cdots \lambda\left(x_{t-1}, x_{t}\right) .
$$

Definition 2.6. A labeling $\lambda$ of $P$ is an EL-labeling if:

1: in each interval $[x, y]$ in $P$, there is a unique saturated chain with a strictly increasing label, and

2: the label of this chain is lexicographically first among the labels of all saturated chains in $[x, y]$.

If $\lambda$ is an EL-labeling of $P$ and each maximal chain is labeled with a permutation of $[d]$ (that is, an element of $\mathcal{S}_{d}$ ) then $\lambda$ is called an $\mathcal{S}_{d}$-EL-labeling.

Example 2.7. The Boolean lattice $B_{d}$ admits an $\mathcal{S}_{d}$-EL-labeling in an obvious way: if $y$ covers $x$ then $y=x \cup\{i\}$, so set $\lambda(x, y)=i$.

When $P$ admits an EL-labeling $\lambda$ and $\mathbf{c}$ is a chain in $P$, we write $\Upsilon_{\lambda}(\mathbf{c})$ to denote the maximal chain of $P$ obtained by filling in each gap in $\mathbf{c}$ with the unique chain in that interval with increasing $\lambda$-label. EL-labelings were introduced by Björner and Wachs, where the following was shown.

Theorem 2.8 ([2]). If $P$ admits an EL-labeling then $\Delta(P)$ is shellable.

For any $S \subseteq[d-1]$ and any maximal chain

$$
\mathbf{c}:=\hat{0}=x_{0}<x_{1}<x_{2}<\cdots<x_{d}=\hat{1}
$$

of $P$, let $\mathbf{c}_{S}$ denote the chain of elements of $\mathbf{c}$ whose ranks lie in $S \cup\{0, d\}$. The rankselected subposet $P_{S}$ is the subposet of $P$ whose maximal chains are all of the form $\mathbf{c}_{S}$, as c ranges over all maximal chains of $P$. Equivalently, $P_{S}$ is the poset $P$ restricted to all elements with ranks in $S \cup\{0, d\}$.

For any $S \subseteq[d-1]$, let $f_{S}$ be the number of maximal chains in $P_{S}$. The collection $\left\{f_{S}\right\}$ is known as the flag f-vector of $P$. Note that the flag $\mathrm{f}$-vector of $P$ refines its f-vector, as clearly

$$
f_{i}(P)=\sum_{S \subseteq[d-1],|S|=i} f_{S}(P) .
$$


The flag h-vector of $P$ is the collection $\left\{h_{S}\right\}$ defined by

$$
h_{S}=\sum_{T \subseteq S}(-1)^{|S-T|} f_{T} .
$$

By inclusion-exclusion, the above is equivalent to $f_{T}=\sum_{S \subseteq T} h_{S}$. It follows that the $\mathrm{h}$-vector is refined by the flag h-vector, namely

$$
h_{i}(P)=\sum_{S \subseteq[d-1],|S|=i} h_{S}(P) .
$$

When $P$ has an EL-labeling, its flag h-vector has a nice enumerative interpretation.

Theorem 2.9 ([2]). The flag h-vector $\left\{h_{S}\right\}$ of a poset $P$ with EL-labeling $\lambda$ is given as follows: $h_{S}$ counts the number of maximal chains of $P$ whose $\lambda$-labels have descent set $S$.

\subsection{Dominance in $\mathcal{S}_{d}$.}

Let $\sigma$ be a permutation in the symmetric group $\mathcal{S}_{d}$. We view $\sigma$ as a word in $[d]$, writing $\sigma=\sigma(1) \sigma(2) \cdots \sigma(d)$. If $\sigma(i)<\sigma(i+1)$ call the interchanging of $\sigma(i)$ and $\sigma(i+1)$ in $\sigma$ a switch.

Recall that the weak order on $\mathcal{S}_{d}$, for which we write $<_{w}$, is the partial order given by the following property: $\sigma<_{w} \tau$ if and only if $\tau$ can be obtained from $\sigma$ by a sequence of switches.

For $S \subseteq[d-1]$, let $D_{S}^{d}$ denote the set of permutation in $\mathcal{S}_{d}$ whose descent sets equal $S:$

$$
\sigma \in D_{S}^{d} \Leftrightarrow\{i: \sigma(i)>\sigma(i+1)\}=S .
$$

Definition 2.10. Let $S, T \subseteq[d-1]$. We say that $S$ dominates $T$ if there exists an injection $\phi: D_{T}^{d} \rightarrow D_{S}^{d}$ such that $\sigma<_{w} \phi(\sigma)$ for all $\sigma \in D_{T}^{d}$.

For example, let $d=4$. Then the set $\{1,3\}$ dominates the set $\{1\}$ via the map

$$
\sigma(1) \sigma(2) \sigma(3) \sigma(4) \rightarrow \sigma(1) \sigma(2) \sigma(4) \sigma(3) .
$$

For a further discussion of dominance in the symmetric group, see [9] or [5].

\section{A decomposition theorem}

The goal of this section is to prove the following theorem, which we will apply in Sections 4 and 5 .

Theorem 3.1. Let $P$ be a rank-d poset with $a \hat{0}$ and $a \hat{1}$, and suppose $P_{1}, P_{2}, \ldots, P_{r}$ are subposets of $P$ satisfying the following properties.

1: Each $P_{i}$ is isomorphic to the Boolean lattice $B_{d}$. 
2: Every chain in $P$ is a chain in some $P_{i}$. Equivalently,

$$
\Delta(P)=\bigcup_{i=1}^{r} \Delta\left(P_{i}\right)
$$

3: Each $P_{i}$ has an $\mathcal{S}_{d}$-EL-labeling $\lambda_{i}$ with the following property: if $\boldsymbol{e}$ is a chain in $P_{i}$ that is also a chain in some $P_{j}$ for $j<i$, then $\Upsilon_{\lambda_{i}}(\boldsymbol{e})$ is a chain in $P_{j}$ for some $j<i$.

Then $\Delta\left(P_{S}\right)$ admits a convex-ear decomposition for any $S \subseteq[d-1]$.

The proof of this theorem relies heavily on our work from [11], where a c.e.d. of $\Delta\left(\left(B_{d}\right)_{S}\right)$ was given for any $S \subseteq[d-1]$. We now review some results from [11] which will be helpful in proving Theorem 3.1.

Fix some $S \subseteq[d-1]$ and an $\mathcal{S}_{d}$-EL-labeling $\lambda$ of $B_{d}$. Let $\mathbf{d}_{1}, \mathbf{d}_{2}, \ldots, \mathbf{d}_{t}$ be all maximal chains in $B_{d}$ whose $\lambda$-labels have descent set $S$, written in lexicographic order of their $\lambda$-labels. For each $i$, let $L_{i}$ be the subposet of $\left(B_{d}\right)_{S}$ generated by the set of maximal chains

$$
\left\{\mathbf{c}_{S}: \mathbf{c} \text { is a maximal chain of } B_{d} \text { with } \mathbf{c}_{[d-1] \backslash S}=\left(\mathbf{d}_{i}\right)_{[d-1] \backslash S}\right\}
$$

Finally, let $\Gamma_{i}$ be the simplicial complex with facets given by maximal chains in $L_{i}$ that are not chains in $L_{j}$ for any $j<i$.

Theorem $3.2([11])$. The sequence of complexes $\Gamma_{1}, \Gamma_{2}, \ldots, \Gamma_{t}$ is a convex-ear decomposition of $\Delta\left(\left(B_{d}\right)_{S}\right)$.

The following lemmata, whose proofs we omit, are shown in [11].

Lemma 3.3. Let $\boldsymbol{e}$ be a maximal chain of some $L_{i}$. Then $\boldsymbol{e}$ is a facet of $\Gamma_{i}$ if and only if

$$
\left(\Upsilon_{\lambda}(\boldsymbol{e})\right)_{[d-1] \backslash S}=\left(\boldsymbol{d}_{i}\right)_{[d-1] \backslash S} .
$$

Lemma 3.4. Let $\boldsymbol{c}$ be the unique maximal chain of $B_{d}$ with increasing $\lambda$-label. Then

$$
\Upsilon_{\lambda}\left(\left(\boldsymbol{d}_{1}\right)_{[d-1] \backslash S}\right)=\boldsymbol{c} .
$$

Lemma 3.5. Let $\boldsymbol{e}_{1}, \boldsymbol{e}_{2}, \ldots, \boldsymbol{e}_{m}$ be all maximal chains corresponding to facets of $\Gamma_{i}$, ordered so that $\lambda\left(\Upsilon_{\lambda}\left(\boldsymbol{e}_{k}\right)\right)$ lexicographically precedes $\lambda\left(\Upsilon_{\lambda}\left(\boldsymbol{e}_{j}\right)\right)$ whenever $j<k$. Then for all $j$ and $k$ with $j<k$, there exists a $j^{\prime}<k$ satisfying

$$
\boldsymbol{e}_{j} \cap \boldsymbol{e}_{k} \subseteq \boldsymbol{e}_{j^{\prime}} \cap \boldsymbol{e}_{k}=\boldsymbol{e}_{j^{\prime}}-x
$$

for some element $x$ of $\boldsymbol{e}_{j^{\prime}}$.

Lemma 3.5, together with Theorem 2.2 and Definition 2.1, proves that $\Gamma_{i}$ is a topological ball for $i>1$. We are now ready to prove our main theorem. 
Proof of Theorem 3.1. The basic idea is to iterate the decomposition provided by Theorem 3.2. Indeed, Theorem 3.2 gives us a c.e.d. of $\Delta\left(\left(P_{1}\right)_{S}\right)$. Now suppose we have a c.e.d. for $X=\bigcup_{i=1}^{q-1} \Delta\left(\left(P_{i}\right)_{S}\right)$ for some $q$ with $2 \leq q \leq r$. We show that we can extend this to a c.e.d. of $X \cup \Delta\left(\left(P_{q}\right)_{S}\right)$. For ease of notation, let $\lambda=\lambda_{q}$ and $\Upsilon=\Upsilon_{\lambda}$.

Taking our cue from the decomposition of $\left(B_{d}\right)_{S}$ described above, let $\mathbf{d}_{1}, \mathbf{d}_{2}, \ldots, \mathbf{d}_{t}$ be all maximal chains of $P_{q}$ whose $\lambda$-labels have descent set $S$, and let the above order be such that $\lambda\left(\mathbf{d}_{i}\right)$ lexicographically precedes $\lambda\left(\mathbf{d}_{j}\right)$ for $i<j$. For each $i$, define $L_{i}$ and $\Gamma_{i}$ as in Theorem 3.2. Finally, let $\Sigma_{i}$ be the simplicial complex whose facets are maximal chains in $\Gamma_{i}$ that are not chains in $X$. We claim that the sequence $\Sigma_{1}, \Sigma_{2}, \ldots, \Sigma_{t}$ (once we remove all $\Sigma_{i}=\emptyset$ ) extends the c.e.d. of $X$. We prove each property of Definition 2.3 separately.

By definition, each $\Gamma_{i} \subseteq \Sigma_{i} \cup X$. Since $\bigcup_{i=1}^{t} \Gamma_{i}=\Delta\left(\left(P_{q}\right)_{s}\right)$ (by Theorem 3.2), $X \cup$ $\left(\bigcup_{i=1}^{t} \Sigma_{i}\right)=X \cup \Delta\left(\left(P_{q}\right)_{S}\right)$, so property $(i)$ holds.

Property $(i i)$ is easily verified as well. Since each $\Gamma_{i}$ for $i>1$ is a proper subcomplex of a simplicial polytope boundary, so is each $\Sigma_{i} \subseteq \Gamma_{i}$. However, as $\Gamma_{1}$ is a simplicial polytope boundary, we need to show that the inclusion $\Sigma_{1} \subseteq \Gamma_{1}$ is proper. This follows from Lemma 3.4, which says that $\mathbf{c}_{S}$ is a facet of $\Gamma_{1}$, where $\mathbf{c}$ is the unique maximal chain in $P_{q}$ with increasing $\lambda$-label. Because $\mathbf{c}=\Upsilon(\hat{0}<\hat{1})$ and $\hat{0}<\hat{1}$ is a chain in all $P_{i}$, it follows that $\mathbf{c}_{S}$ is not a facet of $\Sigma_{1}$.

Now fix $i \geq 1$. To prove property (iii), we employ the techniques (and notation) of Lemma 3.5. Let $\mathbf{e}_{1}, \mathbf{e}_{2}, \ldots, \mathbf{e}_{m}$ be all maximal chains of $\Sigma_{i}$, ordered so that $\lambda\left(\Upsilon\left(\mathbf{e}_{k}\right)\right)$ precedes $\lambda\left(\Upsilon\left(\mathbf{e}_{j}\right)\right)$ whenever $j<k$. Now choose some $j$ and $k$ with $j<k$. Because $\Sigma_{i} \subseteq \Gamma_{i}$, Lemma 3.5 produces a maximal chain $\mathbf{e}$ in $\Gamma_{i}$ satisfying $\mathbf{e}_{j} \cap \mathbf{e}_{k} \subseteq \mathbf{e} \cap \mathbf{e}_{k}=\mathbf{e}-x$ for some element $x$ of $\mathbf{e}$, with $\lambda\left(\Upsilon\left(\mathbf{e}_{k}\right)\right)$ lexicographically preceding $\lambda(\Upsilon(\mathbf{e}))$. To finish the proof, we just need to show that $\mathbf{e}$ is a facet of $\Sigma_{i}$. That is, we need to show that $\mathbf{e}$ is not a chain in $X$.

By Lemma 3.3, $\mathbf{e} \cap \mathbf{e}_{k}=\mathbf{e}-x$ implies that $\Upsilon(\mathbf{e}) \cap \Upsilon\left(\mathbf{e}_{k}\right)=\Upsilon(\mathbf{e})-x$. Because $P_{q}$ is a Boolean lattice, it has exactly two maximal chains containing $\Upsilon(\mathbf{e})-x$ as a subchain. Hence, these chains must be $\Upsilon(\mathbf{e})$ and $\Upsilon\left(\mathbf{e}_{k}\right)$. Because $\lambda\left(\Upsilon\left(\mathbf{e}_{k}\right)\right)$ precedes $\lambda(\Upsilon(\mathbf{e}))$, we have $\Upsilon\left(\mathbf{e}_{k}\right)=\Upsilon(\Upsilon(\mathbf{e})-x)$. If $\mathbf{e}$ were a chain in $X$, then $\Upsilon(\mathbf{e})-x$ would be as well. But then, since $\mathbf{e}_{k}$ is a subchain of $\Upsilon(\Upsilon(\mathbf{e})-x)$, we would have that $\mathbf{e}_{k}$ is in $X$, a contradiction.

For property $(i v)$ consider some $i$, and note that a chain $\mathbf{e}$ is in $\partial \Sigma_{i}$ if and only if there exist two maximal chains $\mathbf{e}_{\text {old }}$ and $\mathbf{e}_{\text {new }}$, each containing $\mathbf{e}$ as a subchain, such that $\mathbf{e}_{\text {old }}$ is a chain in $X \cup\left(\bigcup_{j=1}^{i-1} \Sigma_{j}\right)$ and $\mathbf{e}_{\text {new }}$ is a chain in $\Sigma_{i}$. Thus,

$$
\partial \Sigma_{i} \subseteq \Sigma_{i} \cap\left(X \cup\left(\bigcup_{j=1}^{i-1} \Sigma_{j}\right)\right)
$$

To prove the reverse inclusion, let e be a non-maximal chain in $\Sigma_{i} \cap\left(X \cup\left(\bigcup_{j=1}^{i-1} \Sigma_{j}\right)\right)$. Then by definition e must be a subchain of some maximal chain in $\Sigma_{i}$, and we can take this chain to be $\mathbf{e}_{\text {new }}$. To find $\mathbf{e}_{\text {old }}$, we consider two cases. First, if $\mathbf{e}$ is a chain of $\Sigma_{j}$ for some $j<i$, then by Theorem 3.2 there must be some maximal chain $\mathbf{e}_{\text {old }}$ of $\Sigma_{j}$ for some $j<i$. Second, if $\mathbf{e}$ is a chain in $X$, then $\Upsilon(\mathbf{e})$ must be in $X$ as well. Setting $\mathbf{e}_{\text {old }}=(\Upsilon(\mathbf{e}))_{S}$ 
completes the proof of property $(i v)$.

Thus, we can extend the c.e.d. of $X$ to one of $X \cup \Delta\left(\left(P_{q}\right)_{S}\right)$. Continuing in this fashion, we get a c.e.d. of

$$
\bigcup_{i=1}^{r} \Delta\left(\left(P_{i}\right)_{S}\right) .
$$

By hypothesis every chain in $P$ is a chain in some $P_{i}$ and the above union equals $\Delta\left(P_{S}\right)$, proving the theorem.

\section{Rank-selected geometric lattices}

We first apply Theorem 3.1 to geometric lattices. We assume a basic familiarity with matroid theory, including the cryptomorphism between matroids and geometric lattices. For background, see [1] or [10].

Let $P$ be a rank- $d$ geometric lattice. In [9], Nyman and Swartz show that $\Delta(P)$ admits a convex-ear decomposition. We open this section by briefly describing their technique.

Let $a_{1}, a_{2}, \ldots, a_{\ell}$ be a fixed linear ordering of the atoms of $P$. The minimal labeling $\nu$ of $P$ is defined as follows: if $y$ covers $x$, then $\nu(x, y)=\min \left\{i: x \vee a_{i}=y\right\}$. We view $P$ as the lattice of flats of a simple matroid $M$.

Lemma 4.1 ([1]). The minimal labeling $\nu$ is an EL-labeling.

Lemma $4.2([1])$. Suppose the $\nu$-label of a maximal chain $\boldsymbol{c}$ of $P$ is a word in some subset $B \subseteq\left\{a_{1}, a_{2}, \ldots, a_{\ell}\right\}$. Then $B$ is an nbc-basis of $M$.

Now let $B_{1}, B_{2}, \ldots, B_{t}$ be all the nbc-bases of $M$ listed in lexicographic order. Fix some $j \leq t$ and let $B_{j}=\left\{a_{i_{1}}, a_{i_{2}}, \ldots, a_{i_{d}}\right\}$ with $i_{1}<i_{2}<\cdots<i_{d}$. For a permutation $\sigma \in \mathcal{S}_{d}$, define a maximal chain $\mathbf{c}_{\sigma}^{j}$ of $P$ by

$$
\mathbf{c}_{\sigma}^{j}:=\hat{0}<a_{i_{\sigma(1)}}<\left(a_{i_{\sigma(1)}} \vee a_{i_{\sigma(2)}}\right)<\ldots<\left(a_{i_{\sigma(1)}} \vee a_{i_{\sigma(2)}} \vee \ldots \vee a_{i_{\sigma(d)}}\right)
$$

The basis labeling $\lambda_{j}\left(\mathbf{c}_{\sigma}^{j}\right)$ of $\mathbf{c}_{\sigma}^{j}$ is the word $i_{\sigma(1)} i_{\sigma(2)} \ldots i_{\sigma(d)}$.

For each $i$ with $1 \leq i \leq t$, let $P_{i}$ be the subposet of $P$ whose set of maximal chains is $\left\{\mathbf{c}_{\sigma}^{i}: \sigma \in \mathcal{S}_{d}\right\}$ and let $\Sigma_{i}$ be the simplicial complex whose facets are maximal chains in $P_{i}$ that are not chains in $P_{j}$ for any $j<i$.

Theorem $4.3([9]) . \Sigma_{1}, \Sigma_{2}, \ldots, \Sigma_{t}$ is a convex-ear decomposition of $\Delta(P)$.

The next lemma, shown in [9], is the key tool in proving the above theorem.

Lemma 4.4. A chain $\boldsymbol{c}$ in $P_{i}$ is in $\Sigma_{i}$ if and only if

$$
\lambda_{i}(\boldsymbol{c})=\nu(\boldsymbol{c}) .
$$

The main theorem of this section is the following: 
Theorem 4.5. Let $P$ be a rank-d geometric lattice. Then $\Delta\left(P_{S}\right)$ admits a convex-ear decomposition for any $S \subseteq[d-1]$.

Proof. We show that $P$ satisfies the hypotheses of Theorem 3.1, proving each of the three properties separately.

First note that each $P_{i}$ is isomorphic to the Boolean lattice $B_{d}$ under the mapping $a_{i_{\sigma(1)}} \vee a_{i_{\sigma(2)}} \vee \ldots \vee a_{i_{\sigma(m)}} \rightarrow\{\sigma(1), \sigma(2), \ldots, \sigma(m)\}$, and so property (1) holds. Moreover, the basis labeling $\lambda_{i}$ is an $\mathcal{S}_{d}$-EL-labeling of $P_{i}$ (though with the alphabet $\left\{i_{1}, i_{2}, \ldots, i_{d}\right\}$ rather than $[d]$ ).

By Lemma 4.2, the $\nu$-label of any maximal chain $\mathbf{c}$ is a word in some nbc-basis (say $B_{i}$ ). Thus $\mathbf{c}$ is a chain in $P_{i}$, meaning property (2) holds.

To show property (3), fix some $i$ and suppose that $\mathbf{e}$ is a non-maximal chain in $P_{i}$ that is also a chain in $P_{j}$ for some $j<i$. Suppose that $j$ is the least such integer, and consider the maximal chain $\mathbf{c}=\Upsilon_{\lambda_{j}}(\mathbf{e})$. This chain can clearly not be in $L_{k}$ for any $k<j$, because then $\mathbf{e}$ would be a chain in $L_{k}$, contradicting the minimality of $j$. Thus $\lambda_{j}(\mathbf{c})=\nu(\mathbf{c})$ by Lemma 4.4, meaning $\mathbf{c}=\Upsilon_{\nu}(\mathbf{e})$. Now consider the chain $\mathbf{c}^{\prime}=\Upsilon_{\lambda_{i}}(\mathbf{e})$. If $\mathbf{c}^{\prime}$ is not a chain in $L_{k}$ for any $k<i$ then, again by Lemma $4.4, \nu\left(\mathbf{c}^{\prime}\right)=\lambda_{i}\left(\mathbf{c}^{\prime}\right)$. But then $\mathbf{c}^{\prime}=\Upsilon_{\nu}(\mathbf{e})$, which is a contradiction since the chain $\Upsilon_{\nu}(\mathbf{e})$ is uniquely determined. Thus $\Upsilon_{\lambda_{i}}(\mathbf{e})$ must be a chain in $L_{k}$ for some $k<i$. Applying Theorem 3.1 completes the proof.

\section{Rank-selected face posets}

The main result of this section can be seen as motivated by Hibi's result ([7]) that the codimension-1 skeleton of a shellable complex $\Sigma$ is 2-Cohen-Macaulay. For a simplicial complex $\Sigma$ we write $P_{\Sigma}$ to mean its face poset, the poset of all faces of $\Sigma$ ordered by inclusion. Note that $P_{\Sigma}$ usually does not have a unique maximal element, but the notion of the rank-selected subposet $\left(P_{\Sigma}\right)_{S}$ easily generalizes.

Theorem 5.1. Let $\Sigma$ be a $(d-1)$-dimensional shellable complex. Then $\Delta\left(\left(P_{\Sigma}\right)_{S}\right)$ admits a convex-ear decomposition for any $S \subseteq[d-1]$.

Proof. We wish to apply Theorem 3.1 but, as noted above, a slight adjustment is needed: unless $\Sigma$ consists of a single facet, $P_{\Sigma}$ has no maximal element. To this end, let $P$ be the poset $P_{\Sigma}$ with all its maximal elements identified. As usual, let $\hat{1}$ denote the maximal element of $P$. Clearly, for any $S \subseteq[d-1]$,

$$
\Delta\left(P_{S}\right)=\Delta\left(\left(P_{\Sigma}\right)_{S}\right) .
$$

So, it suffices to apply Theorem 3.1 to $P$. Fix a shelling $F_{1}, F_{2}, \ldots, F_{r}$ of $\Sigma$, and for each $i$ let $P_{i}$ be the face poset of $F_{i}$ (but with its maximal element $F_{i}$ replaced with $\hat{1}$, the maximal element of $P$ ). We claim that the sequence $P_{1}, P_{2}, \ldots P_{r}$ satisfies the hypotheses of Theorem 3.1. Property (1) follows immediately, as the face poset of a $(d-1)$-dimensional simplex is isomorphic to $B_{d}$.

For property (2), let $\mathbf{c}$ be a maximal chain of $P$, and let $x$ be its element of rank $d-1$. Then $x$ is a face of some facet $F_{i}$, meaning $P_{i}$ contains the chain $\mathbf{c}$.

The proof of property (3) relies on the following fact, whose proof is immediate. 
Fact 5.2. Let $\boldsymbol{e}$ be a non-maximal chain in some $P_{i}$, and let $x$ be its element of highest rank $\neq d$. Then $\boldsymbol{e}$ is not a chain in $P_{j}$ for any $j<i$ if and only if, when viewed as a face of $F_{i}$, x contains the unique minimal new face $r\left(F_{i}\right)$.

Now fix some $i$, and let $V$ be the set of vertices of the facet $F_{i}$. Any bijection $\phi: V \rightarrow[d]$ induces an $\mathcal{S}_{d}$-EL-labeling $\lambda_{\phi}$ of $P_{i}$ in the obvious way: For $x, y \in P_{i}$ with $y=x \cup\{v\}$ for some vertex $v$ of $F_{i}$, set $\lambda_{\phi}(x, y)=\phi(v)$ (if $y=\hat{1}$, let $\lambda_{\phi}(x, y)$ be the sole vertex in $\left.V \backslash x\right)$. Let $\phi: F_{i} \rightarrow[d]$ be any bijection that labels vertices in $r\left(F_{i}\right)$ last. That is, if $v \in r\left(F_{i}\right)$ and $w \in F_{i} \backslash r\left(F_{i}\right)$ then $\phi(w)<\phi(v)$. Set $\lambda_{i}=\lambda_{\phi}$. Now suppose $\mathbf{e}$ is a non-maximal chain in $P_{i}$ that is also a chain in $P_{j}$ for some $j<i$, and let $x$ be the element of $\mathbf{e}$ of highest rank $\neq d$. By Fact 5.2,r(Fi) $\nsubseteq x$. If $v$ is the vertex in $F_{i} \backslash x$ with the greatest $\phi$-label then, by definition of $\phi, v \in r\left(F_{i}\right)$. Letting $y$ be the element of $\Upsilon_{\lambda_{i}}(\mathbf{e})$ of rank $d-1$, it follows that $v \notin y$. So $\Upsilon_{\lambda_{i}}(\mathbf{e})$ is a chain in $P_{k}$ for some $k<i$, and property (3) holds.

In many cases, the above theorem does not hold if $d \in S$. For example, if $\Sigma$ is the shellable complex consisting of two 2-dimensional simplices joined at a common boundary facet and $S=\{2,3\} \subseteq[3]$, then $\Delta\left(\left(P_{\Sigma}\right)_{S}\right)$ does not admit a c.e.d., as it is a tree. We can, however, conjecture the following.

Conjecture 5.3. When $\Sigma$ is a $(d-1)$-dimensional complex admitting a convex-ear decomposition and $S \subseteq[d]$, the complex $\Delta\left(\left(P_{\Sigma}\right)_{S}\right)$ admits a convex-ear decomposition.

If $d \notin S$, the above conjecture follows from Theorem 5.1, so we need only consider cases where $d \in S$.

Example 5.4. Let $\Delta$ be a triangulation of the dunce cap, and let $P_{\Delta}$ be its face poset. Then $\left(P_{\Delta}\right)_{S}$ admits a convex-ear decomposition for any $S \subseteq[3]$ with $|S|=2$. To see this, note that a simple graph (viewed as a 1-dimensional simplicial complex) has a c.e.d. if and only if it is 2-connected.

It is well known that $\Delta$ is Cohen-Macaulay, and it is easy to see that $\Delta$ is not 2Cohen-Macaulay. Thus, the converse to Theorem 5.1 is false.

Now recall that a $(d-1)$-dimensional complex $\Sigma$ with vertex set $V$ is called balanced if there exists a $\psi: V \rightarrow[d]$ such that $\psi(v) \neq \psi(w)$ whenever $v$ and $w$ are in a common face of $\Sigma$. The function $\psi$ is called a coloring of $\Sigma$.

The order complex of any graded poset $P$ is always balanced: For a vertex $v$ of $\Delta(P)$, simply let $\psi(v)$ be the rank of $v$ when considered as an element of the poset $P$. Thus the barycentric subdivision of any simplicial complex is balanced, since it is the order complex of its face poset.

If $\Sigma$ is a $(d-1)$-dimensional balanced complex with coloring $\psi$ and $S \subseteq[d]$, define $\Sigma_{S}$ to be the subcomplex of $\Sigma$ with faces $\{F \in \Sigma: \psi(v) \in S$ for all $v \in F\}$. With these new definitions, we can rephrase Theorem 5.1 in a more geometric tone.

Corollary 5.5. Let $\Sigma^{\prime}$ be a $(d-1)$-dimensional shellable complex, and let $\Sigma$ be the first barycentric subdivision of its codimension-1 skeleton. Then, for any coloring $\psi$ of the vertices of $\Sigma$ and any $S \subseteq[d-1]$, the complex $\Sigma_{S}$ admits a convex-ear decomposition. 


\section{The flag h-vector of a face poset}

Our goal in this section is to prove an analogue of the following theorem, shown in [9], for face posets of Cohen-Macaulay complexes. In this section, we assume a basic working knowledge of the Stanley-Reisner ring of a simplicial complex and its Hilbert series (see $[13])$.

Theorem 6.1 ([9]). Let $L$ be a rank-d geometric lattice, and let $S, T \subseteq[d-1]$. If $S$ dominates $T$, the flag $h$-vector of $\Delta(L)$ satisfies $h_{T} \leq h_{S}$.

We state the main theorem now, but postpone its proof until after Theorem 6.5.

Theorem 6.2. Let $K$ be a d-dimensional Cohen-Macaulay simplicial complex with face poset $P$, and let $\Delta=\Delta(P)$. Let $S, T \subseteq[d-1]$, and suppose that $S$ dominates $T$. Then the flag h-vector of $\Delta$ satisfies $h_{T} \leq h_{S}$.

Lemma 6.3. Let $P$ be a rank-d poset with a $\hat{0}$ and $\hat{1}$ whose order complex $\Delta=\Delta(P)$ is a ball. Let $\Delta^{\prime}$ be the set of faces in $\Delta-\partial \Delta-\emptyset$, let $\left\{f_{S}^{\prime}\right\}$ be the flag f-vector of $\Delta^{\prime}$, and let $\left\{h_{S}\right\}$ be the flag h-vector of $\Delta$. Then

$$
\sum_{S \subseteq[d-1]} f_{S}^{\prime} \prod_{i \notin S}\left(\nu_{i}-1\right)=\sum_{S \subseteq[d-1]} h_{[d-1]-S} \prod_{i \notin S} \nu_{i}
$$

Proof. Under the fine grading of the face ring $k[\Delta], F(k[\Delta], \lambda)=\sum_{F \in \Delta} \prod_{x_{i} \in F} \frac{\lambda_{i}}{1-\lambda_{i}}$. We specialize this grading to accommodate the flag h-vector as follows: identify $\lambda_{i}$ and $\lambda_{j}$ whenever the vertices in $\Delta$ to which they correspond have the same rank $r$ (as elements of $P$ ). Call this new variable $\nu_{r}$. This specialized grading yields:

$$
F(k[\Delta], \nu)=\sum_{S \subseteq[d-1]} f_{S} \prod_{i \in S} \frac{\nu_{i}}{1-\nu_{i}}
$$

We put this over the common denominator of $\prod_{i \in[d-1]}\left(1-\nu_{i}\right)$ to obtain:

$$
F(k[\Delta], \nu)=\sum_{S \subseteq[d-1]} \frac{f_{S} \prod_{i \in S} \nu_{i} \prod_{i \notin S}\left(1-\nu_{i}\right)}{\prod_{i \in[d-1]}\left(1-\nu_{i}\right)}=\sum_{S \subseteq[d-1]} \frac{h_{S} \prod_{i \in S} \nu_{i}}{\prod_{i \in[d-1]}\left(1-\nu_{i}\right)}
$$

The following equation is Corollary II.7.2 from [13] (note that $\Delta$ is $(d-2)$-dimensional):

$$
(-1)^{d-1} F(k[\Delta], 1 / \lambda)=(-1)^{d-2} \tilde{\chi}(\Delta)+\sum_{F \in \Delta^{\prime}} \prod_{x_{i} \in F} \frac{\lambda_{i}}{1-\lambda_{i}}
$$

Noting that $\tilde{\chi}(\Delta)=0$, plugging in $1 / \lambda$ in place of $\lambda$, and specializing to the $\nu$-grading, the previous expression becomes:

$$
(-1)^{d-1} F(k[\Delta], \nu)=\sum_{S \subseteq[d-1]} f_{S}^{\prime} \prod_{i \in S} \frac{1}{\nu_{i}-1}
$$


Putting the above over the common denominator of $\prod_{i \in[d-1]}\left(\nu_{i}-1\right)$ and multiplying by $(-1)^{d-1}$ gives us:

$$
F(k[\Delta], \nu)=\sum_{S \subseteq[d-1]} \frac{f_{S}^{\prime} \prod_{i \notin S}\left(\nu_{i}-1\right)}{\prod_{i \in[d-1]}\left(1-\nu_{i}\right)}
$$

Comparing this with Equation (1) and noting that the denominators are equal, we have

$$
\sum_{S \subseteq[d-1]} f_{S}^{\prime} \prod_{i \notin S}\left(\nu_{i}-1\right)=\sum_{S \subseteq[d-1]} h_{S} \prod_{i \in S} \nu_{i}=\sum_{S \subseteq[d-1]} h_{[d-1]-S} \prod_{i \notin S} \nu_{i}
$$

which proves the result.

Now let $\Sigma$ be a $(d-1)$-dimensional shellable complex with face poset $P_{\Sigma}$ and shelling order $F_{1}, F_{2}, \ldots, F_{t}$, and for each $i$ let $P_{i}$ be the face poset of $F_{i}$. Let $A=[d-1]$, and set $\Delta=\Delta\left(\left(P_{\Sigma}\right)_{A}\right)$. Note that $\Delta$ is simply the order complex of $P_{\Sigma}$ once we remove the elements corresponding to the facets of $\Sigma$. Let $\Sigma_{1}, \Sigma_{2}, \ldots, \Sigma_{t}$ be the c.e.d. of $\Delta$ given by Theorem 5.1.

Lemma 6.4. Fix some $\Sigma_{i}$, and let $\lambda_{i}$ be the labeling of $P_{i}$ constructed in the proof of Theorem 5.1. Let $S, T \subseteq[d-1]$, and suppose $S$ dominates $T$. Then there are at least as many maximal chains in $\Sigma_{i}$ whose $\lambda_{i}$-labels have descent set $S$ as chains whose $\lambda_{i}$-labels have descent set $T$.

Proof. Let $\mathbf{c}$ be a maximal chain in $\Sigma_{i}$ with $\sigma=\lambda_{i}(\mathbf{c})$, and let $\sigma(j)<\sigma(j+1)$ be an ascent of its $\lambda_{i}$-label. Let $\mathbf{c}^{\prime}$ is the unique maximal chain of $P_{i}$ that coincides with $\mathbf{c}$ at every rank but $j$, and let $x$ be the element of $\mathbf{c}^{\prime}$ of rank $j$. Then $\mathbf{c}^{\prime}$ must be a chain in $\Sigma_{i}$, since otherwise $\mathbf{c}=\Upsilon_{\lambda_{i}}\left(\mathbf{c}^{\prime}-x\right)$ would not be a chain in $\Sigma_{i}$. So if $\tau$ is a permutation preceded by $\sigma$ in the weak order, there is some maximal chain in $\Sigma_{i}$ with $\tau$ as its $\lambda_{i}$-label. Now suppose $\sigma$ has descent set $T$. If $\phi$ is as in Definition 2.10, it follows that $\Sigma_{i}$ contains a chain whose $\lambda_{i}$-label is $\phi(\sigma)$.

Theorem 6.5. Let $\Sigma$ and $\Delta$ be as above, let $S, T \subseteq[d-1]$, and suppose that $S$ dominates $T$. Then the flag h-vector of $\Delta$ satisfies $h_{T} \leq h_{S}$.

Proof. First, note that $h_{T}\left(\Sigma_{1}\right) \leq h_{S}\left(\Sigma_{1}\right)$, since the poset associated to $\Sigma_{1}$ is just the Boolean lattice $B_{d}$. Now let $\Omega=\Sigma_{1} \cup \Sigma_{2} \cup \cdots \cup \Sigma_{k-1}$ and suppose the result holds for $\Omega$. Let $\Sigma_{k}^{\prime}=\Sigma_{k}-\partial \Sigma_{k}-\emptyset$. Because $\Sigma_{k}$ triangulates a ball, we can now use our earlier expression for the flag h-vector of a ball and invoke an argument similar to Chari's in [3]:

$$
\begin{aligned}
\sum_{S \subseteq[d-1]} h_{S}\left(\Omega \cup \Sigma_{k}\right) \prod_{i \notin S} \nu_{i} & =\sum_{S \subseteq[d-1]} f_{S}\left(\Omega \cup \Sigma_{k}\right) \prod_{i \notin S}\left(\nu_{i}-1\right) \\
& =\sum_{S \subseteq[d-1]} f_{S}(\Omega) \prod_{i \notin S}\left(\nu_{i}-1\right)+\sum_{S \subseteq[d-1]} f_{S}\left(\Sigma_{k}^{\prime}\right) \prod_{i \notin S}\left(\nu_{i}-1\right) \\
& =\sum_{S \subseteq[d-1]} h_{S}(\Omega) \prod_{i \notin S} \nu_{i}+\sum_{S \subseteq[d-1]} h_{[d-1]-S}\left(\Sigma_{k}\right) \prod_{i \notin S} \nu_{i} \text { (by Lemma 6.3) } \\
& =\sum_{S \subseteq[d-1]}\left(h_{S}(\Omega)+h_{[d-1]-S}\left(\Sigma_{k}\right)\right) \prod_{i \notin S} \nu_{i}
\end{aligned}
$$


Thus, for all subsets $S \subseteq[d-1]$,

$$
h_{S}\left(\Omega \cup \Sigma_{k}\right)=h_{S}(\Omega)+h_{[d-1]-S}\left(\Sigma_{k}\right)
$$

Because reverse lexicographic order of the maximal chains of $\Sigma_{k}$ is a shelling (Theorem 3.1), $h_{S}\left(\Sigma_{k}\right)$ counts the number of maximal chains of $\Sigma_{k}$ whose labels have ascent set $S$, and so $h_{[d-1]-S}\left(\Sigma_{k}\right)$ counts the number of maximal chains in $P_{k}$ with descent set $S$. The result now follows from Lemma 6.4.

Proof of Theorem 6.2. Because $P$ is a face poset, a linear inequality of its flag h-vector translates to a linear inequality of the h-vector of $K$. Since every h-vector of a CohenMacaulay complex is the h-vector of some shellable complex (see [13]), the result follows.

We now show that Theorem 6.2 cannot be extended to include posets whose order complexes are Cohen-Macaulay (or 2-CM, for that matter). First recall that a graded poset $P$ is Eulerian if its Möbius function satisfies $\mu(x, y)=(-1)^{\operatorname{rank}(y)-\operatorname{rank}(x)}$ for all $x<y$. An Eulerian poset whose order complex is Cohen-Macaulay is called Gorenstein*. It can be shown that the order complex of a Gorenstein* poset is 2-Cohen-Macaulay. For $S \subseteq[d-1]$, define $w(S)$ to be the set of all $i \in[n]$ such that exactly one of $i$ and $i+1$ is in $S$. For instance, if $S=\{2,3\} \subseteq[4]$ then $w(S)=\{1,3\}$. Since Conjecture 2.3 from [12] was proven by Karu in [8], we can rephrase Proposition 2.8 from [12] as:

Proposition 6.6 ([12]). If $S, T \subseteq[d-1]$ are such that $h_{T}(\Delta) \leq h_{S}(\Delta)$ whenever $\Delta$ is the order complex of a Gorenstein* poset, then $w(T) \subseteq w(S)$.

Now consider $S, T \subseteq[4]$ given by $S=\{1,2\}$ and $T=\{1\}$. In [9], it is shown that $S$ dominates $T$. However, $w(S)=\{2\}$ and $w(T)=\{1\}$, so $w(T) \nsubseteq w(S)$ and it is clear that we cannot extend Theorem 6.2 to include the wider class of Cohen-Macaulay posets (or even 2-CM posets).

Acknowledgements: This paper, which constituted part of the author's Ph.D. thesis, could not have been written without the encouragement, input, and patience of Ed Swartz. Thanks also to the anonymous referee for numerous helpful suggestions.

\section{References}

[1] Anders Björner. The homology and shellability of matroids and geometric lattices. In Matroid applications, volume 40 of Encyclopedia Math. Appl., pages 226-283. Cambridge Univ. Press, Cambridge, 1992.

[2] Anders Björner and Michelle Wachs. On lexicographically shellable posets. Trans. Amer. Math. Soc., 277(1):323-341, 1983.

[3] Manoj K. Chari. Two decompositions in topological combinatorics with applications to matroid complexes. Trans. Amer. Math. Soc., 349(10):3925-3943, 1997. 
[4] Gopal Danaraj and Victor Klee. Shellings of spheres and polytopes. Duke Math. J., 41:443-451, 1974.

[5] Timothy DeVries. The weak order and flag h-vector inequalities. Cornell University senior thesis, 2005.

[6] Patricia Hersh and Ed Swartz. Coloring complexes and arrangements. J. Algebraic Combin., 27(2):205-214, 2008.

[7] Takayuki Hibi. Level rings and algebras with straightening laws. J. Algebra, 117(2):343-362, 1988.

[8] Kalle Karu. The $c d$-index of fans and posets. Compos. Math., 142(3):701-718, 2006.

[9] Kathryn Nyman and Ed Swartz. Inequalities for the $h$-vectors and flag $h$-vectors of geometric lattices. Discrete Comput. Geom., 32(4):533-548, 2004.

[10] James G. Oxley. Matroid theory. Oxford Science Publications. The Clarendon Press Oxford University Press, New York, 1992.

[11] Jay Schweig. A convex-ear decomposition for rank-selected subposets of supersolvable lattices. SIAM J. Discrete Math., 23(2):1009-1022, 2009.

[12] Richard P. Stanley. A survey of Eulerian posets. In Polytopes: abstract, convex and computational (Scarborough, ON, 1993), volume 440 of NATO Adv. Sci. Inst. Ser. C Math. Phys. Sci., pages 301-333. Kluwer Acad. Publ., Dordrecht, 1994.

[13] Richard P. Stanley. Combinatorics and commutative algebra, volume 41 of Progress in Mathematics. Birkhäuser Boston Inc., Boston, MA, second edition, 1996.

[14] Richard P. Stanley. Enumerative combinatorics. Vol. 1, volume 49 of Cambridge Studies in Advanced Mathematics. Cambridge University Press, Cambridge, 1997. With a foreword by Gian-Carlo Rota, Corrected reprint of the 1986 original.

[15] Ed Swartz. g-elements, finite buildings and higher Cohen-Macaulay connectivity. $J$. Combin. Theory Ser. A, 113(7):1305-1320, 2006.

[16] Russ Woodroofe. Cubical convex ear decompositions. Electron. J. Combin., 16(2, Special volume in honor of Anders Bjorner):Research Paper 17, 33, 2009.

[17] Günter M. Ziegler. Lectures on polytopes, volume 152 of Graduate Texts in Mathematics. Springer-Verlag, New York, 1995. 